\title{
Weighted Composition Operators from Derivative Hardy Spaces into $n$-th Weighted-Type Spaces
}

\author{
Nanhui Hu $\mathbb{i D}^{1,2}$ \\ ${ }^{1}$ Department of Mathematics, Shantou University, Shantou 515063, Guangdong, China \\ ${ }^{2}$ Department of Mathematics, Jiaying University, Meizhou 514015, Guangdong, China
}

Correspondence should be addressed to Nanhui Hu; hunhjyu@163.com

Received 15 April 2021; Accepted 3 June 2021; Published 17 June 2021

Academic Editor: Sei Ichiro Ueki

Copyright ( $) 2021$ Nanhui Hu. This is an open access article distributed under the Creative Commons Attribution License, which permits unrestricted use, distribution, and reproduction in any medium, provided the original work is properly cited.

The boundedness, compactness, and the essential norm of weighted composition operators from derivative Hardy spaces into $n$-th weighted-type spaces are investigated in this paper.

\section{Introduction}

Let $H(\mathbb{D})$ denote the space of analytic functions on the open unit disk $\mathbb{D}$. Let $S(\mathbb{D})$ denote the set of all analytic self-maps of $\mathbb{D}$. Let $\varphi \in S(\mathbb{D})$. The composition operator $C_{\varphi}$ with the symbol $\varphi$ is defined by

$$
\left(C_{\varphi} f\right)(z)=f(\varphi(z)), \quad f \in H(\mathbb{D}) .
$$

Let $\varphi \in S(\mathbb{D})$ and $\psi \in H(\mathbb{D})$. The weighted composition operator $\psi C_{\varphi}$ is defined on $H(\mathbb{D})$ by

$$
\left(\psi C_{\varphi} f\right)(z)=\psi(z) f(\varphi(z)), \quad f \in H(\mathbb{D}) .
$$

It is important to give function theoretic descriptions when $\psi$ and $\varphi$ induce a bounded or compact weighted composition operator on various function spaces. See references $[1,2]$ for more information of this research field.

For $0<p<\infty$, the Hardy space, denoted by $\mathscr{H}^{p}$, consists of all functions $f \in H(\mathbb{D})$ such that (see [3])

$$
\|f\|_{\mathscr{H}^{p}}^{p}=\sup _{0<r<1} \frac{1}{2 \pi} \int_{0}^{2 \pi}\left|f\left(r e^{i \theta}\right)\right|^{p} \mathrm{~d} \theta<\infty .
$$

As usual, $\mathscr{H}^{\infty}$ denotes the space of bounded analytic functions in $\mathbb{D}$. If $f^{\prime} \in \mathscr{H}^{p}$, we say that $f$ belongs to the derivative Hardy space, denoted by $\mathcal{S}^{p}$. When $p>1$, the space $\mathcal{S}^{p}$ is a Banach space under the norm defined by

$$
\|f\|_{\mathcal{S}^{p}}^{p}=|f(0)|^{p}+\|f\|_{\mathscr{\ell}^{p}}^{p}
$$

When $p=2, \delta^{2}$ is a Hilbert space. In [4], Roan started the study of composition operators on the space $\mathcal{S}^{p}$. In [5], MacCluer investigated composition operators on the space $\mathcal{S}^{p}$ in terms of Carleson measure. The boundedness and compactness of weighted composition operators on $\mathcal{S}^{p}$ were studied in [6]. See references [4-7] and references therein for more study of composition operators and weighted composition operators on the space $\mathcal{S}^{p}$.

If $\mu$ is a radial, positive, and continuous function on $\mathbb{D}$, then $\mu$ is called a radial weight. Let $\mu$ be a radial weight and $n \in \mathbb{N}$, the set of all positive integers. Let $\mathscr{W}_{\mu}^{n}$ denote the $n$-th weighted space, which consists of all $f \in H(\mathbb{D})$ such that

$$
\|f\|_{\mathscr{W}_{\mu}^{n}}=\sum_{k=0}^{n-1}\left|f^{(k)}(0)\right|+\sup _{z \in \mathbb{D}} \mu(z)\left|f^{(n)}(z)\right|<\infty .
$$

It is a Banach space with the norm $\|\cdot\|_{\mathscr{W}_{\mu}^{n}}$. When $n=1$ and $n=2, \mathscr{W}_{\mu}^{n}$ becomes the Bloch-type space $\mathscr{B}_{\mu}$ and the Zygmund-type space $\mathscr{Z}_{\mu}$, respectively. Furthermore, when $\mu(z)=\left(1-|z|^{2}\right), \mathscr{B}_{\mu}=\mathscr{B}$ is the Bloch space and $\mathscr{Z}_{\mu}=\mathscr{Z}$ is the Zygmund space. For some results on the space $\mathscr{W}_{\mu}^{n}$, see references $[2,8-13]$.

Let $n, k \in \mathbb{N}_{0}$ with $k \leq n$. Recall that the partial Bell polynomials are defined as follows: 


$$
B_{n, k}\left(x_{1}, x_{2}, \ldots, x_{n-k+1}\right)=\sum \frac{n !}{j_{1} ! j_{2} !, \ldots, j_{n-k+1} !}\left(\frac{x_{1}}{1 !}\right)^{j_{1}}\left(\frac{x_{2}}{2 !}\right)^{j_{2}}, \ldots,\left(\frac{x_{n-k+1}}{(n-k+1) !}\right)^{j_{n-k+1}}
$$

where the sum taken over all sequences $j_{1}, j_{2}, \ldots, j_{n-k+1}$ of nonnegative integers such that the following two conditions hold:

$$
\begin{array}{r}
j_{1}+j_{2}+\cdots+j_{n-k+1}=k \\
j_{1}+2 j_{2}+\cdots+(n-k+1) j_{n-k+1}=n .
\end{array}
$$

See reference [14] for more information about Bell polynomials.

In [15], Stević studied the boundedness and compactness of the composition operator from $A_{\alpha}^{p}$ to $\mathscr{W}_{\mu}^{n}$ on the unit disk. In [12], Stević studied the boundedness and compactness of weighted composition operators from $\mathscr{H}^{\infty}$ and the Bloch space to $\mathscr{W}_{\mu}^{n}$. See references [8-10] for more characterizations for weighted composition operators from $\mathscr{H}^{\infty}$ and the Bloch space to $\mathscr{W}_{\mu}^{n}$. In [16], Zhu and Du studied the boundedness, compactness, and essential norm of weighted composition operators from weighted Bergman spaces with doubling weight $A_{\omega}^{p}$ to $\mathscr{W}_{\mu}^{n}$. Recall that the essential norm of a bounded linear operator $T: X \longrightarrow Y$ is its distance to the set of compact operators $K$ mapping $X$ into $Y$, that is,

$$
\|T\|_{e, X \longrightarrow Y}=\inf \left\{\|T-K\|_{X \longrightarrow Y}: K \text { is } a \text { compact operator }\right\} .
$$

Here, $X$ and $Y$ are Banach spaces and $\|\cdot\|_{X \rightarrow Y}$ denotes the operator norm. In [17], Colonna and Tjani studied the boundedness and compactness of weighted composition operators from derivative Hardy spaces $\mathcal{S}^{p}$ to Bloch-type space $\mathscr{B}_{\mu}$.

In this paper, we use Bell polynomials to study the boundedness and compactness of weighted composition operators from derivative Hardy spaces $\mathcal{S}^{p}$ to $n$-th weightedtype spaces $\mathscr{W}_{\mu}^{n}$. Moreover, we give an estimate for the essential norm of weighted composition operators from $\mathcal{S}^{p}$ to $\mathscr{W}_{\mu}^{n}$.

Throughout the paper, we denote by $C$ a positive constant which may differ from one occurrence to the next. In addition, we say that $A \leqslant B$ if there exists a constant $C$ such that $A \leq C B$. The symbol $A \approx B$ means that $A \lesssim B \leq A$.

\section{Boundedness}

In this section, the boundedness of weighted composition operators from $\mathcal{S}^{p}$ to $\mathscr{W}_{\mu}^{n}$ is characterized.

Lemma 1. Suppose $1<p<\infty$ and $k \in \mathbb{N}$. Then, there exists a positive constant $C$ such that

$$
\begin{gathered}
\|f\|_{\infty} \leq C\|f\|_{\mathcal{S}^{p}}, \\
\left|f^{(k)}(z)\right| \leq \frac{C\|f\|_{\mathcal{S}^{p}}}{\left(1-|z|^{2}\right)^{k-1+(1 / p)}}
\end{gathered}
$$

for every $f \in \mathcal{S}^{p}$.

Proof. The first inequality follows from the fact that $\mathcal{S}^{p}$ are contained in the disk algebra for $p>1$. In addition, it is well known that for every $f \in \mathscr{H}^{p}$, there exists a positive constant $C$ such that

$$
\left|f^{(k)}(z)\right| \leq \frac{C\|f\|_{\mathscr{H}^{p}}}{\left(1-|z|^{2}\right)^{k+(1 / p)}}
$$

which implies the second inequality.

For any $a \in \mathbb{D}, 1<p<\infty$, and $j \in\{1,2, \ldots, n+1\}$, set

$$
f_{j, a}(z)=\frac{\left(1-|a|^{2}\right)^{j}}{(1-\bar{a} z)^{j+(1 / p)-1}}, \quad z \in \mathbb{D} \text {. }
$$

After a calculation, for each $j \in\{1,2, \ldots, n+1\}$,

$$
\begin{array}{r}
f_{j, a} \in \mathcal{S}^{p} \\
\sup _{a \in \mathbb{D}}\left\|f_{j, a}\right\|_{\mathcal{S}^{p}}<\infty .
\end{array}
$$

Lemma 2. Let $1<p<\infty$ and $0 \neq a \in \mathbb{D}$. For any $i \in\{1, \ldots, n\}$, there exist constants $c_{2}^{i}, \ldots, c_{n+1}^{i}$ such that

$$
\begin{aligned}
v_{i, a} & =f_{1, a}+\sum_{j=2}^{n+1} c_{j}^{i} f_{j, a} \in \mathcal{S}^{p}, \\
v_{i, a}(a) & =0, \\
v_{i, a}^{(k)}(a) & = \begin{cases}\frac{\bar{a}^{i}}{\left(1-|a|^{2}\right)^{i+(1 / p)-1},} & k=i, \\
0, & k \neq i .\end{cases}
\end{aligned}
$$

Moreover, $v_{i, a}$ converges to 0 uniformly in $\overline{\mathbb{D}}$. 
Proof. The proof is similar to the proof of Theorem 1 in [16]. Hence, we omit the details of the proof.

For the simplicity of this paper, we define

$$
I_{i}^{n}(z)=\sum_{l=i}^{n}\left(\begin{array}{c}
n \\
l
\end{array}\right) \psi^{(n-l)}(z) B_{l, i}\left(\varphi^{\prime}(z), \varphi^{\prime \prime}(z), \ldots, \varphi^{(l-i+1)}(z)\right) .
$$

From the definition, we see that, for example,

$$
\begin{aligned}
B_{0,0}\left(x_{1}\right) & =1, \\
B_{1,0}\left(x_{1}, x_{2}\right) & =0, \\
B_{1,1}\left(x_{1}\right) & =x_{1}, \\
B_{2,0}\left(x_{1}, x_{2}, x_{3}\right) & =0, \\
B_{2,1}\left(x_{1}, x_{2}\right) & =x_{2}, \\
B_{2,2}\left(x_{1}\right) & =x_{1}^{2} .
\end{aligned}
$$

This fact will be used in the proof of the following theorem.

Now, we are in a position to state and prove the first result in this paper.

Theorem 1. Let $n \in \mathbb{N}, 1<p<\infty, \varphi \in S(\mathbb{D}), \psi \in H(\mathbb{D})$, and $\mu$ be a weight. Then, the operator $\psi C_{\varphi}: \mathcal{S}^{p} \longrightarrow \mathscr{W}_{\mu}^{n}$ is bounded if and only if $\psi \in \mathscr{W}_{\mu}^{n}$ and

$$
\sum_{i=1}^{n} \sup _{z \in \mathbb{D}} \frac{\mu(z)\left|\sum_{l=i}^{n}\left(\begin{array}{c}
n \\
l
\end{array}\right) \psi^{(n-l)}(z) B_{l, i}\left(\varphi^{\prime}(z), \varphi^{\prime \prime}(z), \ldots, \varphi^{(l-i+1)}(z)\right)\right|}{\left(1-|\varphi(z)|^{2}\right)^{i+(1 / p)-1}}<\infty
$$

Proof (sufficiency). Let $f \in \mathcal{S}^{p}$. After a calculation, we have (see, e.g., [12])

$$
\left(\psi C_{\varphi} f\right)^{(n)}(z)=\sum_{i=0}^{n} f^{(i)}(\varphi(z)) \sum_{l=i}^{n}\left(\begin{array}{l}
n \\
l
\end{array}\right) \psi^{(n-l)}(z) B_{l, i}\left(\varphi^{\prime}(z), \ldots, \varphi^{(l-i+1)}(z)\right) .
$$

Since $B_{0,0}\left(\varphi^{\prime}(z)\right)=1$ and $B_{l, 0}\left(\varphi^{\prime}(z), \ldots, \varphi^{(l+1)}(z)\right)=$ From (18), for each $j \in\{0,1, \ldots, n-1\}$, $0(l \in \mathbb{N})$, we see that $I_{0}^{n}(z)=\psi^{(n)}(z)$. Hence, by (14) and Lemma 1, we get

$$
\begin{aligned}
& \mu(z)\left|\left(\psi C_{\varphi} f\right)^{(n)}(z)\right| \\
& \leq \mu(z)+\mu(z) \sum_{i=1}^{n}\left|f^{(i)}(\varphi(z))\right|\left|I_{i}^{n}(z)\right|, \\
& \leq\|f\|_{\mathcal{S}^{p}}\|\psi\|_{\mathscr{W}_{\mu}^{n}}+\|f\|_{\mathcal{S}^{p}} \sum_{i=1}^{n} \sup _{z \in \mathbb{D}} \frac{\mu(z)\left|I_{i}^{n}(z)\right|}{\left(1-|\varphi(z)|^{2}\right)^{i+(1 / p)-1}} .
\end{aligned}
$$

$$
\begin{aligned}
\left|\left(\psi C_{\varphi} f\right)^{(j)}(0)\right| & \leq\left|f(\varphi(0))\left\|\psi^{(j)}(0)\left|+\sum_{i=1}^{j}\right| f^{(i)}(\varphi(0))\right\| I_{i}^{j}(0)\right| \\
& \leq\|f\|_{\mathcal{S}^{p}}\left|\psi^{(j)}(0)\right|+\|f\|_{\mathcal{S}^{p}} \sum_{i=1}^{j} \frac{\left|I_{i}^{j}(0)\right|}{\left(1-|\varphi(0)|^{2}\right)^{i+(1 / p)-1}} .
\end{aligned}
$$


Therefore, from (19) and (20) and the fact that $\psi \in \mathscr{W}_{\mu}^{n}$, we get that $\psi C_{\varphi}: \mathcal{S}^{p} \longrightarrow \mathscr{W}_{\mu}^{n}$ is bounded.

Necessity. Assume that $\psi C_{\varphi}: \mathcal{S}^{p} \longrightarrow \mathscr{W}_{\mu}^{n}$ is bounded. It is clear that $\psi \in \mathscr{W}_{\mu}^{n}$, and for each $j \in\{1, \ldots, n+1\}$ and $a \in \mathbb{D}$,

$$
\sup _{a \in \mathbb{D}}\left\|\psi C_{\varphi} f_{j, a}\right\|_{\mathscr{W}_{\mu}^{n}}<\infty
$$

by the boundedness of $\psi C_{\varphi}: \mathcal{S}^{p} \longrightarrow \mathscr{W}_{\mu}^{n}$.

Next, we show that for $i=1,2, \ldots, n$,

$$
\sup _{z \in \mathbb{D}} \mu(z)\left|I_{i}^{n}(z)\right|<\infty \text {. }
$$

Applying the operator $\psi C_{\varphi}$ for $h_{1}(z)=z$, by (17) and (14), we obtain

$$
\begin{aligned}
& \sup _{z \in \mathbb{D}} \mu(z)\left|I_{0}^{n}(z) \varphi(z)+I_{1}^{n}(z)\right|=\sup _{z \in \mathbb{D}} \mu(z)\left|\left(\psi C_{\varphi} h_{1}\right)^{(n)}(z)\right| \\
& \leq\left\|\psi C_{\varphi} h_{1}\right\|_{\mathscr{W}_{\mu}^{n}}<\infty .
\end{aligned}
$$

Therefore, by the triangle inequality and the boundedness of $\varphi$, we obtain

$$
\sup _{z \in \mathbb{D}} \mu(z)\left|I_{1}^{n}(z)\right|<\infty .
$$

Now, we assume that for $1 \leq i \leq j-1(j \leq n)$, $\sup _{z \in \mathbb{D}} \mu(z)\left|I_{i}^{n}(z)\right|<\infty$. To get the desired result, we only need to show that

$$
\sup _{z \in \mathbb{D}} \mu(z)\left|I_{j}^{n}(z)\right|<\infty
$$

Applying the operator $\psi C_{\varphi}$ for $h_{j}(z)=z^{j}$, we obtain

$$
\begin{aligned}
& \sup _{z \in \mathbb{D}} \mu(z)\left|\varphi^{j}(z) I_{0}^{n}(z)+\sum_{k=1}^{j} j(j-1), \ldots,(j-k+1)(\varphi(z))^{j-k}, I_{k}^{n}(z)\right| \\
& \quad \leq\left\|\psi C_{\varphi} h_{j}\right\|_{\mathscr{W}_{\mu}^{n}}<\infty .
\end{aligned}
$$

Hence, from the boundedness of $\varphi$ and triangle inequality again, we get the desired result.
For any $i \in\{1, \ldots, n\}$ and $\varphi(a) \neq 0$, by Lemma 2 ,

$$
\begin{aligned}
\frac{\mu(a)|\varphi(a)|^{i}\left|I_{i}^{n}(a)\right|}{\left(1-|\varphi(a)|^{2}\right)^{i+(1 / p)-1}} & \leq \sup _{a \in \mathbb{D}}\left\|\psi C_{\varphi} v_{i, \varphi(a)}\right\|_{\mathscr{W}_{\mu}^{n}} \\
& \leq \sup _{a \in \mathbb{D}}\left\|\psi C_{\varphi} f_{1, a}\right\|_{\mathscr{W}_{\mu}^{n}}+\sum_{j=2}^{n+1}\left|c_{j}^{i}\right| \sup _{a \in \mathbb{D}}\left\|\psi C_{\varphi} f_{j, a}\right\|_{\mathscr{W}_{\mu}^{n}}<\infty,
\end{aligned}
$$

where $c_{j}^{i}$ are independent of the choice of $a$. From the last inequality and (22), for any $i \in\{1, \ldots, n\}$, we get

$$
\begin{aligned}
& \sup _{|\varphi(a)|>(1 / 2)} \frac{\mu(a)\left|I_{i}^{n}(a)\right|}{\left(1-|\varphi(a)|^{2}\right)^{i+(1 / p)-1}} \\
& \leq \sup _{a \in \mathbb{D}}|| \psi C_{\varphi} f_{1, a}\left\|_{\mathscr{W}_{\mu}^{n}}+\sum_{j=2}^{n+1}\left|c_{j}^{i}\right| \sup _{a \in \mathbb{D}}\right\| \psi C_{\varphi} f_{j, a} \|_{\mathscr{W}_{\mu}^{n}}<\infty, \\
& \sup _{|\varphi(a)| \leq(1 / 2)} \frac{\mu(a)\left|I_{i}^{n}(a)\right|}{\left(1-|\varphi(a)|^{2}\right)^{i+(1 / p)-1}} \lesssim \sup _{|\varphi(a)| \leq(1 / 2)} \mu(a)\left|I_{i}^{n}(a)\right|<\infty .
\end{aligned}
$$

$$
\sum_{i=1}^{n} \sup _{z \in \mathbb{D}} \frac{\mu(z)\left|I_{i}^{n}(z)\right|}{\left(1-|\varphi(z)|^{2}\right)^{i+(1 / p)-1}}<\infty .
$$

The proof is complete.

Let $n=1$. We get the following result, which was first obtained in [17].

Corollary 1. Let $1<p<\infty, \varphi \in S(\mathbb{D}), \psi \in H(\mathbb{D})$, and $\mu$ be a weight. Assume that $\psi C_{\varphi}: \mathcal{S}^{p} \longrightarrow \mathscr{B}_{\mu}$ is bounded if and only if $\psi \in \mathscr{B}_{\mu}$ and

$$
\sup _{z \in \mathbb{D}} \frac{\mu(z)\left|\psi(z) \varphi^{\prime}(z)\right|}{\left(1-|\varphi(z)|^{2}\right)^{(1 / p)}}<\infty .
$$


Let $n=2$. We get the following result.

Corollary 2. Let $1<p<\infty, \varphi \in S(\mathbb{D}), \psi \in H(\mathbb{D})$, and $\mu$ be a weight. Then, the operator $\psi C_{\varphi}: \mathcal{S}^{p} \longrightarrow \mathscr{Z}_{\mu}$ is bounded if and only if $\psi \in \mathscr{Z}_{\mu}$,

$$
\begin{gathered}
\sup _{z \in \mathbb{D}} \frac{\mu(z)\left|\psi(z) \varphi^{\prime \prime}(z)+2 \psi \prime(z) \varphi^{\prime}(z)\right|}{\left(1-|\varphi(z)|^{2}\right)^{(1 / p)}}<\infty \\
\sup _{z \in \mathbb{D}} \frac{\mu(z)\left|\psi(z) \varphi^{\prime 2}(z)\right|}{\left(1-|\varphi(z)|^{2}\right)^{1+(1 / p)}}<\infty .
\end{gathered}
$$

\section{Essential Norm}

In this section, we obtain some estimates for the essential norm of the weighted composition operator $\psi C_{\varphi}: \mathcal{S}^{p} \longrightarrow \mathscr{W}_{\mu}^{n}$, and we need the following lemma.

Lemma 3 (see [17]). Let $X$ be a Banach space that is continuously contained in the disk algebra, and let $Y$ be any Banach space of analytic functions on $\mathbb{D}$. Suppose that

(1) The point evaluation functionals on $Y$ are continuous

(2) For every sequence $\left\{f_{n}\right\}$ in the unit ball of $X$, there exists $f \in X$ and a subsequence $\left\{f_{n_{j}}\right\}$ such that $f_{n_{j}} \longrightarrow f$ uniformly on $\overline{\mathbb{D}}$

(3) The operator $T: X \longrightarrow Y$ is continuous if $X$ has the supremum norm and $Y$ is given the topology of uniform convergence on compact sets

Then, $T$ is a compact operator if and only if, given a bounded sequence $\left\{f_{n}\right\}$ in $X$ such that $f_{n} \longrightarrow 0$ uniformly on $\overline{\mathbb{D}}$, then $\backslash\left\|T f_{n}\right\|_{Y} \longrightarrow 0$ as $n \longrightarrow \infty$.
Lemma 4 (see [17]). Let $1<p<\infty$. Every sequence in $\mathcal{S}^{p}$ bounded in norm has a subsequence which converges uniformly in $\overline{\mathbb{D}}$ to a function in $\mathcal{S}^{p}$.

The following result is a direct consequence of Lemma 3 and Lemma 4.

Lemma 5. Let $n \in \mathbb{N}, 1<p<\infty$, and $\mu$ be a weight. If $T$ is a bounded linear operator from $\mathcal{S}^{p}$ into $\mathscr{W}_{\mu}^{n}$, then $T$ is compact if and only if $\left\|T f_{n}\right\|_{\mathscr{W}^{n}} \longrightarrow 0$ as $n \longrightarrow \infty$ for any sequence $\left\{f_{n}\right\}$ in $\mathcal{S}^{p}$ bounded in norm which convergences to 0 uniformly in $\overline{\mathbb{D}}$.

Theorem 2. Let $n \in \mathbb{N}, 1<p<\infty, \varphi \in S(\mathbb{D}), \psi \in H(\mathbb{D})$, and $\mu$ be a weight such that $\psi C_{\varphi}: \mathcal{S}^{p} \longrightarrow \mathscr{W}_{\mu}^{n}$ is bounded. Then,

$$
\left\|\psi C_{\varphi}\right\|_{e, \mathcal{S}^{p} \longrightarrow \mathscr{W}_{\mu}^{n}} \approx \sum_{i=1}^{n} \limsup _{|\varphi(z)| \longrightarrow 1} \frac{\mu(z)\left|I_{i}^{n}(z)\right|}{\left(1-|\varphi(z)|^{2}\right)^{i+(1 / p)-1}} .
$$

Proof. First, we prove that

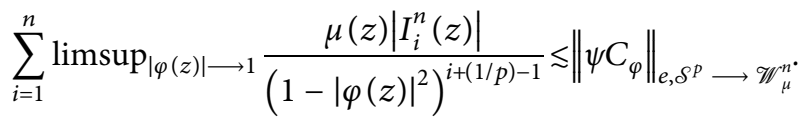

If $\sup _{z \in \mathbb{D}}|\varphi(z)|<1$, there is a number $\delta \in(0,1)$ such that $\sup _{z \in \mathbb{D}}|\varphi(z)|<\delta$. In this case, (32) is vacuously satisfied and the desired result follows.

Assume that $\sup _{z \in \mathbb{D}}|\varphi(z)|=1$. Let $\left\{z_{j}\right\}_{j \in \mathbb{N}}$ be a sequence in $\mathbb{D}$ such that $\left|\varphi\left(z_{j}\right)\right| \longrightarrow 1$ as $j \in \mathbb{N} \longrightarrow$. Since $\psi C_{\varphi}: \mathcal{S}^{p} \longrightarrow \mathscr{W}_{\mu}^{n}$ is bounded, for any compact operator $K: \mathcal{S}^{p} \longrightarrow \mathscr{W}_{\mu}^{n}$ and $i \in\{1, \ldots, n\}$, by using Lemma 2 and Lemma 5, we obtain

$$
\begin{aligned}
\left\|\psi C_{\varphi}-K\right\|_{\mathcal{S}^{p} \longrightarrow \mathscr{W}_{\mu}^{n}} & \geq \limsup _{j \rightarrow \infty}\left\|\psi C_{\varphi} v_{i, \varphi}\left(z_{j}\right)\right\|_{\mathscr{W}_{\mu}^{n}}-\limsup _{j \rightarrow \infty}\left\|K v_{i, \varphi}\left(z_{j}\right)\right\|_{\mathscr{W}_{\mu}^{n}} \\
& \geq \limsup _{j \longrightarrow \infty} \frac{\mu\left(z_{j}\right)\left|\varphi\left(z_{j}\right)\right|^{i}\left|I_{i}^{n}\left(z_{j}\right)\right|}{\left(1-\left|\varphi\left(z_{j}\right)\right|^{2}\right)^{i+(1 / p)-1}}
\end{aligned}
$$

Hence,

$$
\left\|\psi C_{\varphi}\right\|_{e, \mathcal{S}^{p} \longrightarrow \mathscr{W}_{\mu}^{n}} \geq \limsup _{j \longrightarrow \infty} \frac{\mu\left(z_{j}\right)\left|\varphi\left(z_{j}\right)\right|^{i}\left|I_{i}^{n}\left(z_{j}\right)\right|}{\left(1-\left|\varphi\left(z_{j}\right)\right|^{2}\right)^{i+(1 / p)-1}}
$$

which implies that

$$
\left\|\psi C_{\varphi}\right\|_{e, \delta^{p} \longrightarrow \mathscr{W}_{\mu}^{n}} \geq \limsup _{\varphi(z) \longrightarrow 1} \frac{\mu(z)\left|I_{i}^{n}(z)\right|}{\left(1-|\varphi(z)|^{2}\right)^{i+(1 / p)-1}},
$$

as desired.

Now, we show that 
$\left\|\psi C_{\varphi}\right\|_{e, \mathcal{S}^{p} \longrightarrow \mathscr{W}_{\mu}^{n}} \lesssim \sum_{i=1}^{n} \limsup _{|\varphi(z)| \longrightarrow 1} \frac{\mu(z)\left|I_{i}^{n}(z)\right|}{\left(1-|\varphi(z)|^{2}\right)^{i+(1 / p)-1}}$.

Let $r \in[0,1)$ and define $K_{r} f(z)=f_{r}(z)=f(r z)$. Then, $K_{r}: \mathcal{S}^{p} \longrightarrow \mathcal{S}^{p}$ is compact and $\left\|K_{r}\right\|_{\mathcal{S}^{p} \longrightarrow \mathcal{S}^{p}} \leq 1$. It is clear that $f_{r} \longrightarrow f$ uniformly on compact subsets of $\mathbb{D}$ as $r \longrightarrow 1$. Let $\left\{r_{j}\right\} \subset(0,1)$ be a sequence such that $r_{j} \longrightarrow 1$ as $j \longrightarrow \infty$. Then, for any positive integer $j$, the operator $\psi C_{\varphi} K_{r_{j}}: \mathcal{S}^{p} \longrightarrow \mathscr{W}_{\mu}^{n}$ is compact. By the definition of the essential norm, we get

$$
\left\|\psi C_{\varphi}\right\|_{e, \mathcal{S}^{p} \longrightarrow \mathscr{W}_{\mu}^{n}} \leq \limsup _{j \longrightarrow \infty}\left\|\psi C_{\varphi}-\psi C_{\varphi} K_{r_{j}}\right\|_{\mathcal{S}^{p} \longrightarrow \mathscr{W}_{\mu}^{n}} .
$$

Hence, it is sufficient to show that

$$
\limsup _{j \longrightarrow \infty}\left\|\psi C_{\varphi}-\psi C_{\varphi} K_{r_{j}}\right\|_{\mathcal{S}^{p} \longrightarrow \mathscr{W}_{\mu}^{n}} \leqslant \sum_{i=1}^{n} \limsup _{|\varphi(z)| \longrightarrow 1} \frac{\mu(z)\left|I_{i}^{n}(z)\right|}{\left(1-|\varphi(z)|^{2}\right)^{i+(1 / p)-1}}
$$

For any $f \in \mathcal{S}^{p}$ such that $\|f\|_{\mathcal{S}^{p}} \leq 1$,

$$
\begin{aligned}
& \left\|\left(\psi C_{\varphi}-\psi C_{\varphi} K_{r_{j}}\right) f\right\|_{\mathscr{W}_{\mu}^{n}} \\
& \leq \sum_{t=0}^{n-1} \sum_{i=0}^{t}\left|\left(f-f_{r_{j}}\right)^{(i)}(\varphi(0))\right|\left|I_{i}^{t}(0)\right|+\sup _{z \in \mathbb{D}} \mu(z) \sum_{i=0}^{n}\left|\left(f-f_{r_{j}}\right)^{(i)}(\varphi(z)) \| I_{i}^{n}(z)\right| \\
& \leq \underbrace{\sum_{t=0}^{n-1} \sum_{i=0}^{t}\left|\left(f-f_{r_{j}}\right)^{(i)}(\varphi(0)) I_{i}^{t}(0)\right|}_{\Omega_{0}}+\underbrace{\sup _{z \in \mathbb{D}} \mu(z)\left|\left(f-f_{r_{j}}\right)(z)\right|\left|I_{0}^{n}(z)\right|}_{\Omega_{1}} \\
& +\underbrace{\sup _{|\varphi(z)| \leq r_{N}} \mu(z) \sum_{i=1}^{n}\left|\left(f-f_{r_{j}}\right)^{(i)}(\varphi(z)) \| I_{i}^{n}(z)\right|}_{\Omega_{2}} \\
& +\underbrace{\sup _{|\varphi(z)|>r_{N}} \mu(z) \sum_{i=1}^{n}\left|\left(f-f_{r_{j}}\right)^{(i)}(\varphi(z)) \| I_{i}^{n}(z)\right|}_{\Omega_{3}}
\end{aligned}
$$

where $N \in \mathbb{N}$ such that $r_{j} \geq(2 / 3)$ for all $j \geq N$. Since for any nonnegative integer $s,\left(f-f_{r_{j}}\right)^{(s)} \longrightarrow 0$ uniformly on compact subsets of $\mathbb{D}$ as $j \longrightarrow \infty$. It is clear that

$$
\begin{aligned}
& \limsup _{j \longrightarrow \infty} \Omega_{0}=0 \\
& \limsup _{j \longrightarrow \infty} \Omega_{2}=0 .
\end{aligned}
$$

From Lemma 4,

$\lim _{j \rightarrow \infty} \Omega_{1} \leq\|\psi\|_{\mathscr{W}_{\mu}^{n}} \lim _{j \rightarrow \infty} \sup _{z \in \mathbb{D}}\left|\left(f-f_{r_{j}}\right)(z)\right|=0$,

while

$$
\Omega_{3} \leq \sum_{i=1}^{n} \underbrace{\sup _{|\varphi(z)|>r_{N}} \mu(z)\left|f^{(i)}(\varphi(z)) \| I_{i}^{n}(z)\right|_{P_{i}}+\sum_{i=1}^{n} \underbrace{\sup _{|\varphi(z)|>r_{N}} \mu(z)\left|r_{j}^{i} f^{(i)}\left(r_{j} \varphi(z)\right) \| I_{i}^{n}(z)\right|_{Q_{i}}}}
$$

For any $i \in\{1, \ldots, n\}$, by Lemma 1 and Lemma 2 , 


$$
\begin{aligned}
& P_{i}=\sup _{|\varphi(z)|>r_{N}} \mu(z) \frac{\left(1-|\varphi(z)|^{2}\right)^{i+(1 / p)-1}\left|f^{(i)}(\varphi(z))\right|}{|\varphi(z)|^{i}} \frac{|\varphi(z)|^{i}\left|I_{i}^{n}(z)\right|}{\left(1-|\varphi(z)|^{2}\right)^{i+(1 / p)-1}} \\
& \quad \leq\|f\|_{\mathcal{S}^{p}} \sup _{|\varphi(z)|>r_{N}}\left\|\psi C_{\varphi} v_{i, \varphi(z)}\right\|_{\mathscr{W}_{\mu}^{n}} \\
& \quad \lesssim \sup _{|a|>r_{N}}\left\|\psi C_{\varphi} f_{1, a}\right\|_{\mathscr{W}_{\mu}^{n}}+\sum_{k=2}^{n+1}\left|c_{k}^{i}\right| \sup _{|a|>r_{N}}\left\|\psi C_{\varphi} f_{k, a}\right\|_{\mathscr{W}_{\mu}^{n}} \\
& \quad \lesssim \sum_{k=1}^{n+1} \sup _{|a|>r_{N}}\left\|\psi C_{\varphi} f_{k, a}\right\|_{\mathscr{W}_{\mu}^{n} \cdot}
\end{aligned}
$$

Taking the limit as $N \longrightarrow \infty$, by Lemma 1 , we get

$$
\limsup _{j \longrightarrow \infty} P_{i} \leq \limsup _{|\varphi(z)| \longrightarrow 1} \frac{\mu(z)\left|I_{i}^{n}(z)\right|}{\left(1-|\varphi(z)|^{2}\right)^{i+(1 / p)-1}} \text {. }
$$$$
\limsup _{j \longrightarrow \infty} Q_{i} \lesssim \limsup _{|\varphi(z)| \longrightarrow 1} \frac{\mu(z)\left|I_{i}^{n}(z)\right|}{\left(1-|\varphi(z)|^{2}\right)^{i+(1 / p)-1}} .
$$

Hence, by (40)-(43), (45), and (46), we obtain

By the same reason, we have

$$
\begin{aligned}
\limsup _{j \rightarrow \infty} \| & \psi C_{\varphi}-\psi C_{\varphi} K_{r_{j}} \|_{\mathcal{S}^{p} \longrightarrow \mathscr{W}_{\mu}^{n}} \\
& =\limsup _{j \longrightarrow \infty} \sup _{\|f\|_{\delta p} \leq 1}\left\|\left(\psi C_{\varphi}-\psi C_{\varphi} K_{r_{j}}\right) f\right\|_{\mathscr{W}_{\mu}^{n}} \lesssim \sum_{i=1}^{n} \limsup _{|\varphi(z)| \longrightarrow} \frac{\mu(z)\left|I_{i}^{n}(z)\right|}{\left(1-|\varphi(z)|^{2}\right)^{i+(1 / p)-1}}
\end{aligned}
$$

Hence, from the last two inequalities, we obtain (39). The proof is complete.

From Theorem 2 and the well-known result that $\|T\|_{e, X \longrightarrow Y}=0$ if and only if $T: X \longrightarrow Y$ is compact, we obtain the following corollary.

Corollary 3. Let $n \in \mathbb{N}, 1<p<\infty, \varphi \in S(\mathbb{D}), \psi \in H(\mathbb{D})$, and $\mu$ be a weight. Assume that $\psi C_{\varphi}: \mathcal{S}^{p} \longrightarrow \mathscr{W}_{\mu}^{n}$ is bounded, then the operator $\psi C_{\varphi}: \mathcal{S}^{p} \longrightarrow \mathscr{W}_{\mu}^{n}$ is compact if and only if

$$
\sum_{i=1}^{n} \limsup _{|\varphi(z)| \longrightarrow 1} \frac{\mu(z)\left|I_{i}^{n}(z)\right|}{\left(1-|\varphi(z)|^{2}\right)^{i+(1 / p)-1}}=0 .
$$

Let $n=1$. We get the following result, which was first obtained in [17].

Corollary 4. Let $1<p<\infty, \varphi \in S(\mathbb{D}), \psi \in H(\mathbb{D})$, and $\mu$ be a weight such that $\psi C_{\varphi}: \mathcal{S}^{p} \longrightarrow \mathscr{B}_{\mu}$ is bounded. Then, the operator $\psi C_{\varphi}: \mathcal{S}^{p} \longrightarrow \mathscr{B}_{\mu}$ is compact if and only if

$$
\limsup _{|\varphi(z)| \longrightarrow 1} \frac{\mu(z)\left|\psi(z) \varphi^{\prime}(z)\right|}{\left(1-|\varphi(z)|^{2}\right)^{(1 / p)}}=0 .
$$

Let $n=2$. We get the following result.

Corollary 5. Let $1<p<\infty, \varphi \in S(\mathbb{D}), \psi \in H(\mathbb{D})$, and $\mu$ be a weight such that $\psi C_{\varphi}$ : $\mathcal{S}^{p} \longrightarrow \mathscr{Z}_{\mu}$ is bounded. Then, the operator $\psi C_{\varphi}: \mathcal{S}^{p} \longrightarrow \mathscr{Z}_{\mu}$ is compact if and only if

$$
\begin{array}{r}
\limsup _{|\varphi(z)| \longrightarrow 1} \frac{\mu(z)\left|\psi(z) \varphi^{\prime \prime}(z)+2 \psi \prime(z) \varphi^{\prime}(z)\right|}{\left(1-|\varphi(z)|^{2}\right)^{(1 / p)}}=0 \\
\limsup _{|\varphi(z)| \longrightarrow 1} \frac{\mu(z)\left|\psi(z) \varphi^{\prime 2}(z)\right|}{\left(1-|\varphi(z)|^{2}\right)^{1+(1 / p)}}=0 .
\end{array}
$$

\section{Data Availability}

No data were used to support this study.

\section{Conflicts of Interest}

The author declares that there are no conflicts of interest. 


\section{References}

[1] C. Cowen and B. MacCluer, Composition Operators on Spaces of Analytic Functions, CRC Press, Boca Raton, FL, USA, 1995.

[2] K. Zhu, "Operator theory in function spaces," Mathematical Surveys and Monographs, Vol. 138, American Mathematical Society, Providence, RI, USA, 2ed edition, 2007.

[3] P. Duren, Theory of $H^{p}$ Spaces, Academic Press, New York, NY, USA, 1970.

[4] R. Roan, "Composition operators on the space of functions with $H^{p}$-derivative," Houston Journal of Mathematics.vol. 4, pp. $423-438,1978$.

[5] B. Maccluer, "Composition operators on $\mathcal{S}^{p}, "$ Houston Journal of Mathematics.vol. 13, pp. 245-254, 1987.

[6] M. Contreras and A. Hernandez-Diaz, "Weighted composition operators on spaces with derivative in a Hardy space," Journal of Operator Theory, vol. 52, pp. 173-184, 2004.

[7] Q. Lin, J. Liu, and Y. Wu, "Volterra type operators on Sp (D) spaces," Journal of Mathematical Analysis and Applications, vol. 461, no. 2, pp. 1100-1114, 2018.

[8] E. Abbasi, S. Li, and H. Vaezi, "Weighted composition operators from the Bloch space tonth weighted-typespaces," Turkish Journal of Mathematics, vol. 44, no. 1, pp. 108-117, 2020.

[9] E. Abbasi, H. Vaezi, and S. Li, "Essential norm of weighted composition operators from $H^{\infty}$ to $\mathrm{t} n \mathrm{~h}$ weighted type spaces," Mediterranean Journal of Mathematics, vol. 16, no. 5, p. 14, 2019.

[10] S. Li, E. Abbasi, and H. Vaezi, "Weighted composition operators from Bloch-type spaces to $\$ \mathrm{n} \$$ th weighted-type spaces," Annales Polonici Mathematici, vol. 124, no. 1, pp. 93-107, 2020.

[11] S. Stević, "Composition followed by differentiation from $H^{\infty}$ and the Bloch space to $-n$th weighted-type spaces on the unit disk," Applied. Mathematics and Computation, vol. 216, no. 12 , pp. $3450-3458,2010$.

[12] S. Stević, "Weighted differentiation composition operators from $H^{\infty}$ and Bloch spaces to $-n$th weighted-type spaces on the unit disk," Applied. Mathematics and Computation, vol. 216, pp. 3634-3641, Article ID 246287, 2010.

[13] S. Stević, "Essential norm of some extensions of the generalized composition operators between $k$-th weighted-type spaces," Journal of Inequalities and Applications.vol. 2017, p. 220, 2017.

[14] L. Comtet, Advanced Combinatorics, D. Reidel, Dordrecht, Netherlands, 1974.

[15] S. Stević, "Composition operators from weighted Bergman space to $n$-th weighted space on the unit disk," Discrete Dynamics in Nature and Society.vol. 2009, Article ID 742019, 11 pages, 2009.

[16] X. Zhu and J. Du, "Weighted composition operators from weighted Bergman spaces to Stević-type spaces," Mathematical Inequalities \& Applications, vol. 22, no. 1, pp. 361-376, 2019.

[17] F. Colonna and M. Tjani, "Weighted composition operators from Banach spaces of analytic functions into Bloch-type spaces," Problems and Recent Methods in Operator Theory, vol. 687, pp. 75-95, 2017. 